\title{
Efectos de la práctica pianística en la sintomatología postquirúrgica del cáncer de mama Jose Carril Miranda ${ }^{1}$ Francisco Martínez Rodríguez ${ }^{2}$
}

Recibido: 14/09/2018 /Aceptado: 6/11/2018

\section{RESUMEN}

El presente estudio analiza la disminución del dolor postquirúrgico en las mujeres intervenidas de cáncer de mama a través de movimientos ejecutados en la técnica pianística. Durante su realización, ocho mujeres, elegidas aleatoriamente de una muestra total de dieciséis, realizaron distintos ejercicios en un piano durante diez sesiones. Cada mujer del grupo experimental practicó los movimientos pianísticos seleccionados con la extremidad superior homolateral al área de la intervención quirúrgica. El análisis estadístico de los resultados obtenidos reveló un alivio significativo del dolor.

Palabras clave: Dolor, piano, movimiento, cáncer.

\section{Effects of piano practice on postoperative symptomatology of breast cáncer}

\begin{abstract}
The current study analyses the decreasing postoperative pain in woman who had breast cancer surgery through movements carried out in piano techniques. During the implementation period, eigth women chosen randomly from a sample of sixteen, performed several activities with a piano during ten sessions. Each woman in the experimental group, performed the selected piano movements with the upper extermity ipsilateral to the area of the surgical intervention. The statistical analysis of the results obtained, indicate a significant pain relief.
\end{abstract}

Key words: Pain, piano, movement, cancer.

Sumario. 1. Introducción 2. Objetivos.2. Material y métodos 3. Resultados 4. Discusión 5. Conclusiones 6. Referencias

Cómo citar. Carril, J. y Martínez, F. (2018) Efectos de la práctica pianística en la sintomatología postquirúrgica del cáncer de mama. Revista de Investigación en Musicoterapia, 2, 35-46

\footnotetext{
${ }^{1}$ Beca formativa como pianista acompañante en la Escuela Municipal de Música de Oviedo.jose_algator@hotmail.com

${ }^{2}$ Fisioterapeuta en la clínica Tálamo de Avilés. fmrodriguez@hotmail.com
} 


\section{Introducción}

El cáncer de mama, con 26.370 casos nuevos diagnosticados en el año 2017 en España según la Sociedad Española de Oncología Médica, constituye la primera causa de enfermedad por cáncer en la mujer.

Entre los factores de riesgo se encuentran: edad, susceptibilidad genética, exposición hormonal, factores ambientales, tabaco, alcohol, obesidad y sedentarismo.

El cáncer de mama y su tratamiento dejan secuelas físicas y psicológicas en un alto porcentaje de las mujeres que lo padecen. El tratamiento es multidisciplinario e incluye cirugía, radioterapia, quimioterapia, hormonoterapia y terapia molecular, con una tendencia hacia terapias personalizadas y conservadoras. En muchas ocasiones se requiere de un apoyo psicológico debido a la afectación de la vida social y familiar de las personas afectadas.

Los métodos diagnósticos de detección precoz han incrementado sus probabilidades de curación. En algunos casos, en los que la patología no tiene tratamiento curativo, la esperanza de vida se puede prolongar gracias a la continua investigación, que resulta determinante.

En relación con las secuelas físicas y psicológicas destacan, entre otras: dolor postquirúrgico, cambios en la imagen corporal con disminución de la autoestima (Valverde, García-Nietoy Gutiérrez, 2014), inseguridad frente a nuevas posibilidades de recidiva, ansiedad y depresión.

El tratamiento quirúrgico puede producir dolor e impotencia funcional en relación con el área intervenida, dificultando la actividad diaria y laboral. Cuando existe afección ganglionar y se indica el vaciamiento axilar puede producirse un linfedema que ocasiona morbilidad.

Entre las estrategias de afrontamiento del cáncer de mama se incluye la música, que se ha utilizado desde la Antigüedad por ser un buen canal de comunicación (Calle-Albert, Ignacio, 2013) y aliviar dolencias físicas y psicológicas, disminuyendo la ansiedad y el estrés mediante mecanismos como la relajación y la distracción, mejorando la calidad de vida de las pacientes con cáncer (Trallero-Flix, 2004), (Marti, 2015).

Estudios realizados con tomografía por emisión de positrones indican que las respuestas emocionales placenteras ante la música activan estructuras cerebrales 
relacionadas con la compensación, la emoción y la atención, disminuyendo la activación en aquellas áreas relacionadas con episodios aversivos. Mediante resonancia magnética se han comprobado patrones de activación funcional significativos (Haslinger, Erhard, Altenmulleret al., 2004). También se observaron cambios electromiográficos y variaciones en las frecuencias cardiaca y respiratoria. Este hallazgo vincula la música con estímulos biológicamente relevantes (Blood y Zatorre, 2006).

Un elemento más de la música es el movimiento realizado en la ejecución de su técnica. $\mathrm{Y}$ en este aspecto nos hemos centrado en este estudio ya que, a largo plazo, un número significativo de supervivientes de cáncer de mama muestran, además de dolor, alteración de la movilidad del hombro de la parte intervenida (Devoogdt, Van Kampen, Christiaens, Troosters, Piot, Beets, Nys y Gosselink, 2011), por lo que se considera que la aplicación de diversas medidas fisioterapéuticas pueden mejorar la restauración funcional del miembro superior afectado (Gosselink, Rouffaer, Vanhelden, Piot, Troosters y Christiaens, 2003). Los programas de ejercicios deben dirigirse al rango de movimiento y a la corrección de la postura (Shamley, Srinanaganathan, Weatherall, Oskrochi, Watson, Ostlere et al., 2007).

En el presente estudio se han diseñado como herramientas terapéuticas diversos movimientos ejecutados en la técnica pianística. Se ha pretendido valorar si esos movimientos implicados en la producción del sonido pueden actuar como un reforzamiento positivo, de modo que el conjunto de movimientos ejercidos para la activación del teclado a fin de producir el sonido musical requerido pueda aliviar el dolor postquirúrgico crónico de las pacientes intervenidas de cáncer de mama.

El dolor, según la Asociación Mundial para el Estudio del Dolor, "es una experiencia sensorial y emocional desagradable, asociada con un daño tisular, real o potencial, o descrita en términos de dicho daño" (1994). Es un fenómeno subjetivo y es el paciente quien mejor lo define. El dolor típico del paciente con cáncer en ocasiones se escapa al control farmacológico. Es crónico, ilimitado en su duración y frecuentemente está acompañado de componente psicológico (Puebla-Díaz, 2005). Además, las mujeres intervenidas de cáncer de mama pueden presentar dificultades en la movilidad del hombro tras la cirugía (Levangie, Santasier, Stout y Pfalzer, 2011). Inicialmente, lo que más influye en la afectación del movimiento es el proceso de cicatrización, la presencia de drenajes a nivel de la herida quirúrgica y el dolor postoperatorio (García, Cruz, Pérez, Pérez, Manzanero y Cantalapiedra, 2011). Tanto el dolor como la pérdida de 
movilidad del hombro alteran significativamente las actividades cotidianas y la calidad de vida en estos casos (Nesvold, Reinertsen, Fosså y Dahl, 2011).

\section{Objetivos}

El objetivo principal ha sido valorar si la intervención con movimientos propios de la técnica pianística puede producir variaciones en el dolor postquirúrgico del cáncer de mama. El proyecto es de especial interés por la falta de referencias que relacionan el dolor con la interpretación musical.

Este estudio ha pretendido valorar si la interacción entre el movimiento propuesto y la música producida por las pacientes podría generar nuevas estrategias destinadas a disminuir su sufrimiento físico y psicológico, fortalecer los recursos existentes y mejorar su calidad de vida.

Consideramos importante la adquisición de nuevos conocimientos sobre el tema ya que no hemos encontrado ningún trabajo de investigación que relacione la intervención con movimientos propios de la técnica pianística y las variaciones en la sintomatología postquirúrgica.

\section{Material y métodos}

Los criterios de inclusión han sido mujeres intervenidas de cáncer de mama en los 24 meses previos al inicio del estudio que no estuvieran recibiendo tratamiento de quimioterapia ni radioterapia en ese momento (para evitar el fenómeno de aversión condicionada). Los criterios de exclusión fueron discapacidad física o psíquica, trastornos sensoriales auditivos severos, pacientes inmovilizadas y trastorno mental severo.

Las variables recogidas fueron: edad, formación musical, datos relacionados con la patología mamaria, datos relativos al miembro superior relacionado con el área intervenida quirúrgicamente y fecha de intervención. Los datos recogidos relacionados con la patología tratada quirúrgicamente fueron: tipo de tumor, tipo de intervención y tiempo transcurrido entre la intervención y el inicio del estudio.

Inicialmente se seleccionaron 18 pacientes pero hubo dos pérdidas (por incidencias familiares en ambos casos), una en cada grupo, por lo que estos quedaron finalmente equilibrados. La muestra final del presente estudio la constituyeron 16 sujetos (8 asignadas de forma aleatoria al grupo control y 8 al grupo experimental), todas mujeres, 
supervivientes de cáncer de mama, con edades comprendidas entre 26 y 71 años. La media de edad de la muestra fue de 49,6 años.

La mayor parte de las intervenciones quirúrgicas fueron mastectomías. El tipo de tumor más frecuente fue el carcinoma ductal infiltrante. En 8 sujetos el lado afectado fue el izquierdo, en 7 el derecho y en 1 bilateral.

Los datos relativos al miembro superior relacionado con el área intervenida quirúrgicamente fueron los rangos articulares y el balance muscular en flexión, extensión, abducción, aducción, rotación interna y rotación externa; la circometría o medida de perímetro en cinco niveles; y la ergonomía instrumental antes y después de la intervención. Como elementos de medida se completaron los cuestionarios validados de ansiedad (Escala de Hamilton) y dolor (Cuestionario EVA).

El dolor, variable principal de la hipótesis a estudio, se evaluó antes y después de la intervención mediante el Cuestionario EVA, en la que se pidió a las pacientes que marcasen en dicha línea el punto de dolor que consideraran según su intensidad.

El test ofrecido es muy intuitivo ya que refleja una gama de colores que va del blanco (no existe dolor), al rojo intenso (el dolor es muy severo), en un trapecio de color que se apoya en una regla milimetrada sobre una línea horizontal de 10 centímetros.

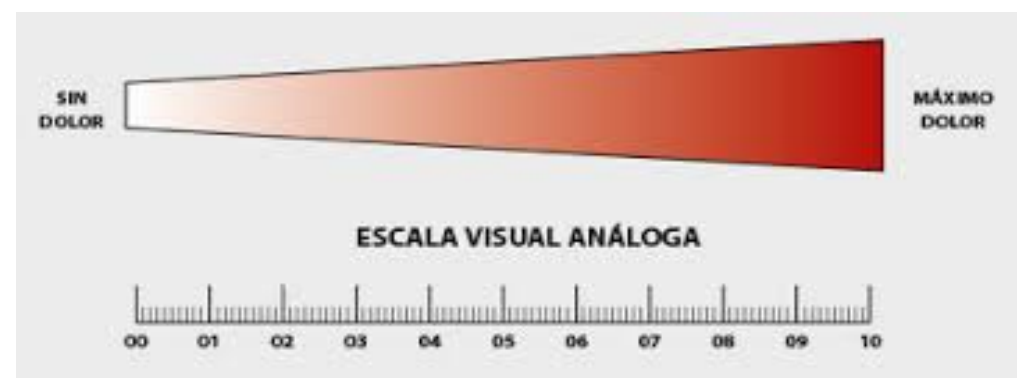

Imagen 1. Cuestionario EVA, utilizado para la evaluación del dolor.

Todas estas variables se recogieron en las hojas de recogida de datos al principio y al final de la intervención.

Metodología: Contraste de una hipótesis previa

La variable dolor (ordinal) es la principalmente estudiada. La hipótesis previa consistía en la convicción de que la práctica de técnica pianística disminuiría el dolor postquirúrgico en cáncer de mama.

\section{Procedimiento}


En el grupo de casos se llevó a cabo la intervención, consistente en 10 sesiones de aplicación de la técnica pianística seleccionada, que tuvo lugar en las instalaciones de la Asociación Española Contra el Cáncer de Mama de Gijón. Las pacientes recibieron la intervención de "movimientos ejecutados mediante técnica pianística" en la extremidad superior homolateral al área intervenida por cáncer de mama.

La práctica en el piano consistió en la interpretación de escalas y acordes a través de patrones de movimientos diseñados "ad hoc", teniendo en cuenta la angulación utilizada en la rehabilitación fisioterapéutica. Se realizaron los tres acordes triada principales de la tonalidad de Do M: tónica, dominante y subdominante. El acorde de tónica se trabajó en estado fundamental (do-mi-sol), el acorde de dominante en primera inversión (si-resol) y el acorde de subdominante en segunda inversión (do-fa-la). Las tres escalas practicadas en la intervención fueron Do M, Sol M y Re M, todas en un rango de dos octavas. La escala de Do M se interpreta utilizando solamente las teclas blancas del piano, la escala de Sol M utiliza una tecla negra (fa\#) y la escala de Re M dos teclas negras (fa\# y do\#).

En el grupo control se registraron todos los datos de las variables al principio y al final del estudio pero no se realizó ninguna intervención.

Se utilizaron las técnicas pianísticas diseñadas a tal fin, movilizando la extremidad superior homolateral al área de intervención, presionando las teclas del piano con la fuerza y la duración adecuadas al sonido deseado, para conseguir un determinado estímulo auditivo con un adecuado rango de movilidad.

El presente estudio, diseñado como un ensayo clínico, fue aprobado por el Comité Ético de Ensayos Clínicos del Principado de Asturias. Se seleccionó a los sujetos que cumplían los criterios de inclusión y se les informó sobre el estudio. Las personas que manifestaron su interés en participar en el mismo firmaron el documento de Consentimiento Informado antes de su inicio.

\section{Resultados}

El análisis estadístico fue realizado con el programa SPSS para Windows. 
Tabla 1

Se muestran las puntuaciones directas obtenidas en el Cuestionario EVA antes y después en el grupo experimental y en el grupo control

\begin{tabular}{|c|c|c|c|}
\hline Casos & Dolor inicial & Dolor final & Grupo \\
\hline 1 & 9,2 & 3,9 & Intervención \\
\hline 2 & 4,6 & 2,8 & Intervención \\
\hline 3 & 0,9 & $\mathbf{0}$ & Intervención \\
\hline 4 & 9 & 1,9 & Intervención \\
\hline 5 & 6,6 & 4,3 & Intervención \\
\hline 6 & 6,8 & 2 & Intervención \\
\hline 7 & 3,3 & 1,8 & Intervención \\
\hline 8 & 5,9 & 1,3 & Intervención \\
\hline 9 & 3,9 & 5,4 & Control \\
\hline 10 & 0,4 & 0,5 & Control \\
\hline 11 & 9 & 5,5 & Control \\
\hline 12 & 5 & 6,5 & Control \\
\hline 13 & 1 & 1,8 & Control \\
\hline 14 & 1 & 3,8 & Control \\
\hline 15 & 0,3 & 5,8 & Control \\
\hline 16 & 7,7 & 6,1 & Control \\
\hline
\end{tabular}

En los siguientes gráficos se representa una comparación del dolor antes y después en el grupo control y en el grupo experimental. En el eje de ordenadas se representa el dolor en escala ordinal y en el eje de abscisas se representan los ocho sujetos de cada grupo mediante números. 


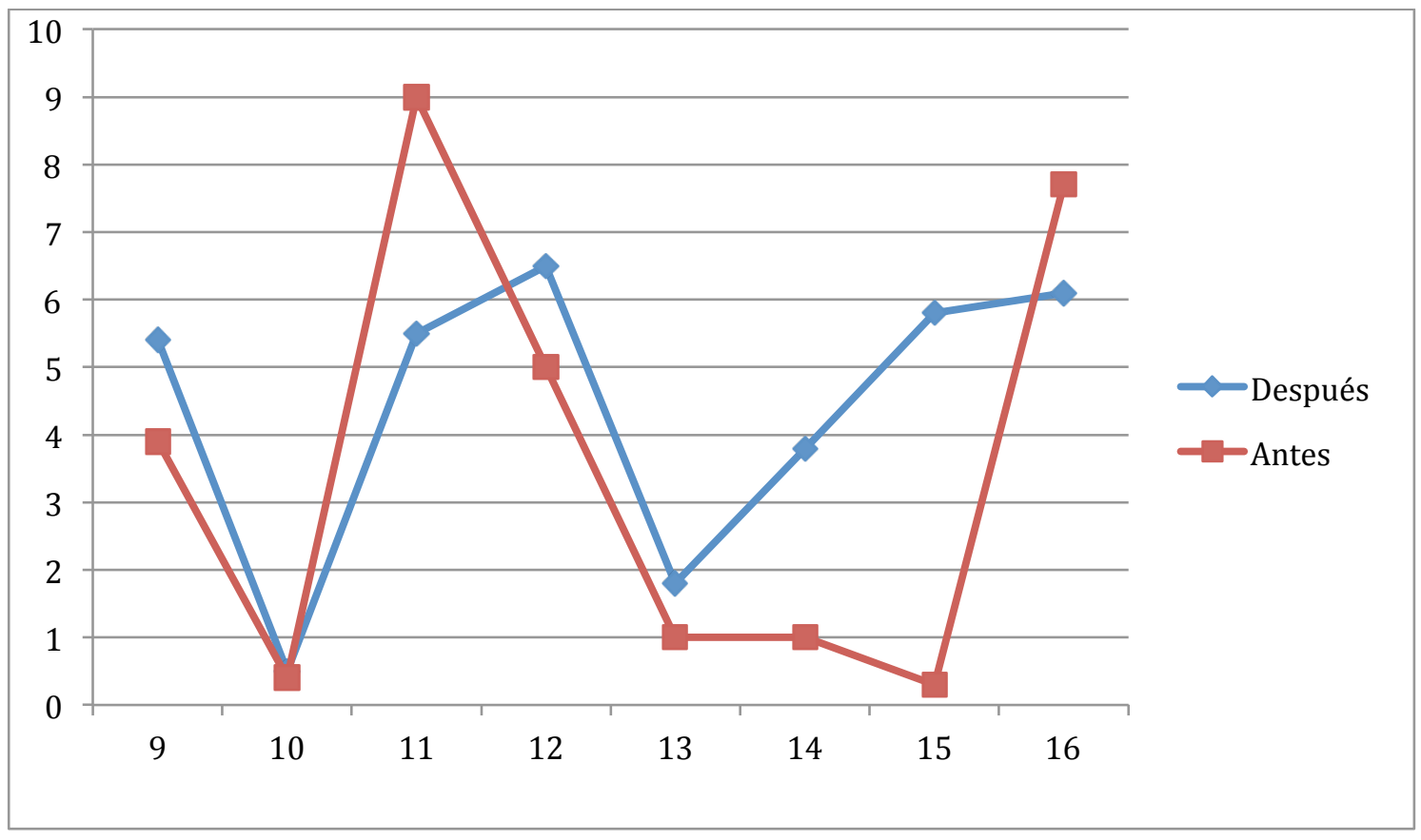

Figura 2. Dolor antes y después en el grupo control

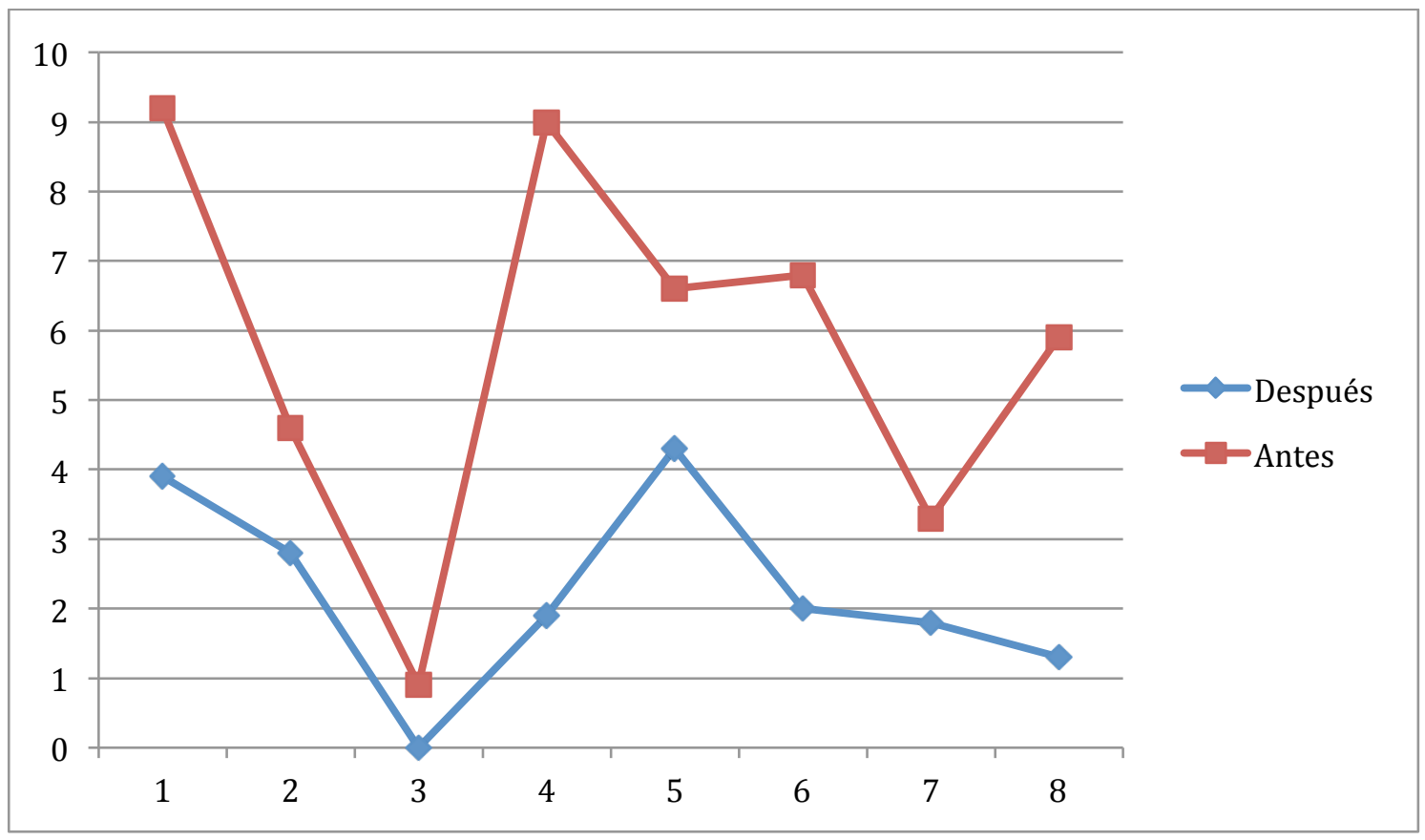

Figura 3. Dolor antes y después en el grupo experimental. Como se puede observar, los sujetos del grupo de casos experimentan una disminución significativa del dolor que no se observa entre los sujetos del grupo control. 


\section{Prueba ANOVA par:}

Condiciones de aplicación:

-Normalidad (Kolmogorov-Smirnov, por haber menos de 30 casos):

- en "dolor antes", $p=0,658$ (se asume que hay normalidad)

- en "dolor después", $p=0,710$ (se asume que hay normalidad)

-Homocedasticidad u homogeneidad de varianzas (prueba de Levene):

- en "dolor antes", $p=0,380$ (se asume que hay homocedasticidad)

- en "dolor después", $p=0,146$ (se asume que hay homocedasticidad)

Tabla 2

Test de ANOVA par

\begin{tabular}{cccccc}
\hline Fuente & Sum.cuadr.III & g de I & Cuad.Med. & F & P \\
\hline (Ant.-Des.) & 14,045 & 1 & 14,045 & 4,566 & 0,051 \\
\hline $\begin{array}{c}\text { (Ant.- } \\
\text { Des.)*Grupo }\end{array}$ & 39,161 & 1 & 39,161 & 12,761 & 0,003 \\
\hline Error & 42,964 & 14 & 3,069 & & \\
\hline
\end{tabular}

Pruebas de contraste dentro de sujetos

Se adopta como nivel de significación global, $\mathrm{p}$ igual o menor de 0,05 . Por lo tanto, la interacción entre el dolor antes-dolor después con el grupo es muy significativa.

Se puede decir que:

- Con la intervención hay mejoría del dolor muy significativa, por lo que también la habrá en la mayoría de la población a la que pertenece la muestra.

- La mejoría es importante (pasa de 5,8 a 2,2 de media).

Tabla 3

Estadísticos descriptivos

\begin{tabular}{lllll}
\hline $0=$ control,1=casos & Media & Desviación estándar & $\mathrm{N}$ \\
\hline Dolor & 0 & 3,538 & 3,4359 & 8 \\
Antes & 1 & 5,788 & 2,8068 & 8 \\
& Total & 4.663 & 3,2459 & 16 \\
\hline
\end{tabular}




\begin{tabular}{lllll}
\hline Dolor & 0 & 4,425 & 2,1972 & 8 \\
Después & 1 & 2,250 & 1,3928 & 8 \\
& Total & 3,338 & 2,1023 & 16 \\
& & & & \\
\hline
\end{tabular}

En la Tabla 3 aparece la media y desviación típica para el grupo control y grupo experimental antes y después de la intervención.

Tabla 4

Medias marginales estimadas

\begin{tabular}{llll}
$\begin{array}{l}0=\text { control } \\
1=\text { caso }\end{array}$ & Factor 1 & Media & $\begin{array}{l}\text { Error } \\
\text { estándar }\end{array}$ \\
\hline 0 & 1 & 3,538 & 1,109 \\
& 2 & 4,425 &, 650 \\
\hline 1 & 1 & 5,788 & 1,109 \\
& 2 & 2,250 &, 650 \\
\hline
\end{tabular}

Medida: tiempo

\section{Discusión}

Los movimientos de la técnica pianística, relacionados con la producción musical, y seleccionados para el presente estudio, han contribuido a la disminución del dolor crónico residual en el grupo de pacientes intervenidas en cáncer de mama que han participado como grupo de casos en este trabajo de investigación. De este modo se puede enunciar que los movimientos de la técnica pianística seleccionados pueden convertirse en un apoyo más para la superación de la sintomatología postquirúrgica de la intervención por cáncer de mama.

En cuanto a la interpretación del análisis estadístico, los resultados son muy significativos. Tratándose de una investigación o estudio confirmatorio (no exploratorio, pues existía una hipótesis previa a la realización de la investigación que ha servido de base para la planificación o estrategia metodológica), las conclusiones son válidas. Como se observa en la última tabla de resultados la media del dolor en el grupo experimental tres puntos y medio, frente al ligero incremento presente en el grupo control. 
La eficacia de este ensayo se basa en que los sujetos experimentales han tenido un papel activo de interpretación y no solo de audición musical. La música como terapia complementaria en el cáncer de mama constituye un elemento que refuerza las estrategias de afrontamiento de las pacientes frente a esta enfermedad.

Como limitación principal del estudio cabe citar la escasa muestra disponible de sujetos que cumplieran los criterios de inclusión.

\section{RECONOCIMIENTOS}

El estudio se ha realizado íntegramente con los recursos humanos y materiales de los investigadores y ha contado con la ayuda inestimable, para la difusión previa a su inicio, del personal de la AECC de Gijón.

Su realización ha sido posible gracias a la Dirección de la AECC en Asturias.

Se agradece la colaboración de $\mathrm{M}^{\mathrm{a}}$ Ángeles Miranda, Alfonso Villa, Margarita Collado, Mabel Núñez y Rocío Toledo.

Los recursos materiales utilizados han sido: piano, partituras, instrumentos de medida, fichas de registro de actividad y sistemas de grabación de audio y vídeo.

\section{Referencias}

Blood A.J., Zatorre R.J. (2006). Intensely pleasurable responses to music correlate with activity in brain regions implicated in reward and emotion. Journal of Music Therapy; 43: 295-316.

Calle-Albert, I. (2013). Historia de la musicoterapia I y II Desde la Edad Media hasta el Prerromanticismo. Cuadernos de Bellas Artes 19 y 20, La Laguna. (Tenerife): Latina.

Devoogdt N, Van Kampen M, Christiaens MR, Troosters T, Piot W, Beets N, Nys S, Gosselink R. (2011). Short- and long-term recovery of upper limb function after axillary lymph node dissection. Eur J Cancer Care (Engl); 20(1):77-86.

García, M. T. F., Cruz, N. E. M., Pérez, F. G., Pérez, C. E., Manzanero, M. Á. P., y Cantalapiedra, J. A., (2011). Programa de ejercicios de miembro superior tras cirugía de cáncer de mama. Bases científicas. SERMEF.

Gosselink R, Rouffaer L, Vanhelden P, Piot W, Troosters T, Christiaens MR. (2003). Recovery of upper limb function after axillary dissection. J Surg Oncol; 83(4):204- 211.

Haslinger, B., Erhard, P., Altenmuller, E., et al. (2004). "Reduced recruitment of motor association areas during bimanual coordination in concert pianists". Hum Brain Mapp; (22):206-215.

Levangie PK, Santasier AM, Stout NL, Pfalzer L. (2011). A qualitative assessment of upper quarter dysfunction reported by physical therapists treated for breast cancer or treating breast cancer sequelae. Support Care Cancer; 19(9):13671378.

Marti A.P. et al. (2015). Efecto de la musicoterapia en el estado anímico y calidad de 
vida de un grupo de mujeres supervivientes de cáncer de mama. Psicooncología; (12): 105-128.

Nesvold IL, Reinertsen KV, Fosså SD, Dahl AA. (2011). The relation between arm/shoulder problems and quality of life in breast cancer survivors: a crosssectional and longitudinal study. J Cancer Surviv; 5(1):62-72.

Puebla-Díaz, F. (2005). Tipos de dolor y escala terapéutica de la OMS. Dolor iatrogénico. Oncología; 28 (3): 139-143.

Segura-Valverde, M., García-Nieto, R., y Gutiérrez, L. A. S. (2014). Imagen corporal y autoestima en mujeres mastectomizadas. Psicooncología; 11(1):45-57.

Shamley DR, Srinanaganathan R, Weatherall R, Oskrochi R, Watson M, Ostlere S, et al. (2007). Changes in shoulder muscle size and activity following treatment for breast cancer. Breast Cancer Res Treat; 106(1):19-27.

Trallero-Flix, C. (2004). Musicoterapia creativa en pacientes con cáncer: un método para disminuir la ansiedad y el estrés. Revista virtual Psiquiatría.com. 